\title{
SEPARABILITY AND ENTANGLEMENT OF FOUR-MODE GAUSSIAN STATES
}

\section{Vladimir I. Man'ko ${ }^{1}$ and Alexandr A. Sergeevich ${ }^{2}$}

\author{
${ }^{1}$ P. N. Lebedev Physical Institute, Russian Academy of Sciences, \\ Leninskii Prospect 53, Moscow 119991, Russia \\ ${ }^{2}$ Moscow Institute of Physics and Technology (State University), \\ Institutskii per. 9, Dolgoprudnyi, Moscow Region 141700, Russia \\ e-mails: manko@sci.lebedev.ru a.sergeevich@gmail.com
}

\begin{abstract}
The known Peres-Horodecki criterion and scaling criterion of separability are considered on examples of three-mode and four-mode Gaussian states of electromagnetic field. It is shown that the principal minors of the photon quadrature dispersion matrix are sensitive to the change of scaling parameters. An empirical observation has shown that the bigger the modulus of negative principal minors, the more entangled the state.
\end{abstract}

Keywords: entanglement, Gaussian states, scaling transform, multimode light, separability criterion.

\section{Introduction}

The entanglement phenomenon is one of the most important quantum properties of multimode Gaussian states (both pure and mixed) was studied in the context of their separability and entanglement in [1, 2] where the Peres-Horodecki criterion of separability [3, 4] was implemented for detecting the entanglement of two-mode-light Gaussian state. The Peres-Horodecki criterion of separability of a quantum state is based on the property of positive but not completely positive map of the density matrix [5], which is the transpose transform of the density matrix. For a separable state of a bipartite system, the partial transpose transform applied to variables of one subsystem yields a new density matrix that corresponds to the physical state. For the density matrix of an entangled state, the partial transpose transform can give a matrix that is a negative Hermitian matrix. The criterion (called ppt-criterion) was used in [1, 2] to study the entanglement 
of Gaussian states of photons. Later on, other researchers successfully applied the ppt-criterion (see, for example, [6-9]).

The other separability criterion based on a nonpositive map of the density matrix, called the partial scaling transform, was suggested in [10, 11]; its implementation is connected with properties of the Wigner function [12] with respect to the scaling of momentum. For a separable state of a two-mode light, the scaling of the second component of momentum provides an admissible Wigner function. The scaling criterion can be extended to the case of multipartite quantum states. The ppt-criterion is a special case of the scaling criterion with the choice of the scaling parameter equal to -1 . The scaling criterion was used recently by Chirkin and Saigin [13, 14] to study the entanglement properties of three-mode light generated in nonlinear crystals due to the interaction, where three different processes are involved, namely, two parametric down-conversions and one parametric up-conversion.

In this work, we study the application of the scaling criterion to three- and four-mode photon states. We consider both the pure and mixed states. Our aim is to find some empirical correlations of the degree of entanglement and negative values of principal minors of the photon quadrature dispersion matrix as functions of the scaling parameters.

The paper is organized as follows.

In Sec. 2 the definition of separability is discussed. In Sec. 3 the Peres-Horodecki criterion is reviewed. The properties of scaling transform are discussed in Sec. 4. The application of the scaling criterion to the three-mode case is elaborated in Sec.5, while the four-mode case is studied for a pure Gaussian photon state in Sec. 6. A mixed four-mode Gaussian state is considered in Sec. 7. Some conclusions and perspectives are presented in Sec. 8.

\section{Separability and Entanglement}

We consider a single-mode photon state described by the quadrature operators $\hat{q}$ and $\hat{p}$. The density matrix $\rho$ of any field state obeys the following conditions:

$$
\rho^{+}=\rho, \quad \operatorname{Tr} \rho=1, \quad \rho \geq 0 .
$$

When taking a transpose of the density matrix, these conditions are kept for a new 'transposed' state:

$$
\left(\rho^{T}\right)^{+}=\rho^{T}, \quad \operatorname{Tr} \rho^{T}=1, \quad \rho^{T} \geq 0 .
$$

The transpose transform of the density matrix is equivalent to the time inversion or complex conjugation of the density matrix. There is a one-to-one correspondence between density operators and Wigner quasidistribution functions $W(q, p)$. From the definition of the Wigner function it follows that the transpose transform of $\rho$ is equivalent to a mirror reflection of momentum $p$ in the phase space, namely,

$$
\rho \longrightarrow \rho^{T} \Longleftrightarrow W(q, p) \longrightarrow W(q,-p)
$$


Now we consider a two-mode state with the density operator $\hat{\rho}(1,2)$ and annihilation operators

$$
\hat{a}_{1}=\frac{\hat{q}_{1}+i \hat{p}_{1}}{\sqrt{2}}, \quad \hat{a}_{2}=\frac{\hat{q}_{2}+i \hat{p}_{2}}{\sqrt{2}} .
$$

We say that a state is simply separable if

$$
\hat{\rho}(1,2)=\hat{\rho}(1) \otimes \hat{\rho}(2) .
$$

A separable two-mode state is defined as a state with the density operator representable as a convex sum of simply separable states:

$$
\hat{\rho}(1,2)=\sum_{j} k_{j} \hat{\rho}_{1}^{(j)}(1) \otimes \hat{\rho}_{2}^{(j)}(2), \quad \sum_{j} k_{j}=1, \quad k_{j} \geq 0 .
$$

Here $\hat{\rho}_{1}^{(j)}(1)$ and $\hat{\rho}_{2}^{(j)}(2)$ are the density operators of modes 1 and 2 , respectively. The states which cannot be presented in such a form are called entangled states. In other words, by definition, an entangled state is a state which cannot be presented as a convex sum of simply separable states.

\section{The Peres-Hrodecki Criterion}

It is evident from (4) that the partial transpose, i.e., the transpose of a matrix for the second-mode term $\rho_{2}^{(j)}(2) \rightarrow\left(\rho_{2}^{(j)}(2)\right)^{T}$, leads to a separable positive density operator

$$
\hat{\rho}^{p t}(1,2)=\sum_{j} k_{j} \hat{\rho}_{1}^{(j)}(1) \otimes\left(\hat{\rho}_{2}^{(j)}(2)\right)^{T} .
$$

Thus, $\hat{\rho}^{p t}(1,2)$ describes a new separable state.

Summarizing, we can say that the partial transpose $\hat{\rho} \rightarrow \hat{\rho}^{p t}$ of a separable state necessarily gives the density operator, which fits the conditions (1). This is the Peres-Horodecki criterion of separability.

Rewriting the decomposition (5) in terms of Wigner functions we obtain

$$
W\left(q_{1}, p_{1}, q_{2}, p_{2}\right)=\sum_{j} l_{j} W_{1}^{(j)}\left(q_{1}, p_{1}\right) W_{2}^{(j)}\left(q_{2}, p_{2}\right) .
$$

Let us consider the Wigner function of the generic Gaussian form

$$
W(q, p)=\frac{1}{\sqrt{\operatorname{det} \sigma}} \exp \left(-\frac{1}{2} \mathbf{Q} \sigma^{-\mathbf{1}} \mathbf{Q}^{\mathbf{T}}\right),
$$

where the four-dimensional vector $\mathbf{Q}$ reads

$$
\mathbf{Q}=\left(q_{1}-\left\langle q_{1}\right\rangle, q_{2}-\left\langle q_{2}\right\rangle, p_{1}-\left\langle p_{1}\right\rangle, p_{2}-\left\langle p_{2}\right\rangle\right)
$$


and the matrix $\sigma$ is a $4 \times 4$ real symmetric variance matrix

$$
\sigma_{r_{i} r_{j}}=\frac{1}{2}\left\langle\hat{r}_{i} \hat{r}_{j}+\hat{r}_{j} \hat{r}_{i}\right\rangle
$$

with $\hat{r}_{1}=\hat{q}_{1}, \hat{r}_{2}=\hat{q}_{2}, \hat{r}_{3}=\hat{p}_{1}, \hat{r}_{4}=\hat{p}_{2}$.

From (3) and (8) it is easy to see that the partial transpose is equivalent to the transform of a variance matrix $\sigma \rightarrow \sigma^{\prime}$, which is defined as follows:

$$
\left(\begin{array}{cccc}
\sigma_{q_{1} q_{1}} & \sigma_{q_{1} q_{2}} & \sigma_{q_{1} p_{1}} & \sigma_{q_{1} p_{2}} \\
\sigma_{q_{2} q_{1}} & \sigma_{q_{2} q_{2}} & \sigma_{q_{2} p_{1}} & \sigma_{q_{2} p_{2}} \\
\sigma_{p_{1} q_{1}} & \sigma_{p_{1} q_{2}} & \sigma_{p_{1} p_{1}} & \sigma_{p_{1} p_{2}} \\
\sigma_{p_{2} q_{1}} & \sigma_{p_{2} q_{2}} & \sigma_{p_{2} p_{1}} & \sigma_{p_{2} p_{2}}
\end{array}\right) \longrightarrow\left(\begin{array}{cccc}
\sigma_{q_{1} q_{1}} & \sigma_{q_{1} q_{2}} & \sigma_{q_{1} p_{1}} & -\sigma_{q_{1} p_{2}} \\
\sigma_{q_{2} q_{1}} & \sigma_{q_{2} q_{2}} & \sigma_{q_{2} p_{1}} & -\sigma_{q_{2} p_{2}} \\
\sigma_{p_{1} q_{1}} & \sigma_{p_{1} q_{2}} & \sigma_{p_{1} p_{1}} & -\sigma_{p_{1} p_{2}} \\
-\sigma_{p_{2} q_{1}} & -\sigma_{p_{2} q_{2}} & -\sigma_{p_{2} p_{1}} & \sigma_{p_{2} p_{2}}
\end{array}\right)
$$

The conditions of existence of a partial transposed state in the Gaussian case can be rewritten in the form of the Robertson-Schrödinger uncertainty relation [15, 16]. Thus, the Peres-Horodecki criterion of separability is equivalent to the condition of positivity of principal minors of the matrix

$$
\Sigma=\sigma^{\prime}+\frac{i}{2} \Omega
$$

where

$$
\Omega=\left(\begin{array}{cccc}
0 & 0 & -1 & 0 \\
0 & 0 & 0 & -1 \\
1 & 0 & 0 & 0 \\
0 & 1 & 0 & 0
\end{array}\right)
$$

Here the dimensionless units with $\hbar=1$ are used.

Since the transform considered does not change the minors but the determinant, the PeresHorodecki criterion can be simplified into the inequality

$$
\operatorname{det} \Sigma \geq 0 \text {. }
$$

This criterion is a necessary and sufficient condition of separability of Gaussian states in the two-mode case, but the application of the Peres-Horodecki criterion to the $n$-mode case with $n \geq 3$ fails to detect all entangled states.

\section{Scaling Transform}

Now we consider a scaling transform, which is applicable for detecting the entanglement of Gaussian states. We generalize the reflection transform $p \rightarrow-p$ in the phase space and use the scaling of momentum $p \rightarrow \lambda p$. In that way, we define the map

$$
\left\{\begin{array}{l}
q \longrightarrow q, \\
p \longrightarrow \lambda p .
\end{array}\right.
$$


For $\lambda \in[-1,1]$, this map defines a semigroup of maps. The map (15) is equivalent to the scaling of time $t \rightarrow \lambda t$. In the case $\lambda=1$, this is an identical map; for $\lambda=-1$ we have a partial transpose used in the Peres-Horodecki criterion of separability, which was discussed in the previous section.

Upon applying the scaling transform, the single-mode Wigner function $W(q, p)$ modifies as follows:

$$
W(q, p) \longrightarrow W_{\lambda}(q, p)=N W(q, \lambda p),
$$

where $N$ is a normalization constant.

The initial Wigner function is normalized

$$
\int W(q, p) \frac{d q d p}{2 \pi}=1
$$

and the transformed Wigner function must be normalized, as well:

$$
\int W_{\lambda}(q, p) \frac{d q d p}{2 \pi}=\int N W(q, \lambda p) \frac{d q d p}{2 \pi}=1 .
$$

Substituting $p^{\prime}=\lambda p$ into the integral (18), we obtain

$$
\int N W\left(q, p^{\prime}\right) \frac{d q d p^{\prime}}{2 \pi|\lambda|}=1
$$

Taking into account (16) we have the following result for the normalization constant $N$ :

$$
\frac{N}{|\lambda|}=1 \Longrightarrow N=|\lambda| \text {. }
$$

Hence, the scaling transform modifies the Wigner function as follows:

$$
W(q, p) \longrightarrow W_{\lambda}(q, p)=|\lambda| W(q, \lambda p) .
$$

Now we find out how the partial scaling transform affects the dispersion matrix of the twomode quantum state. We perform the scaling of the second momentum by the factor of $\lambda$,

$$
p_{2} \longrightarrow \lambda p_{2}
$$

Obviously, the variance matrix $\sigma$ converts into

$$
\sigma_{\lambda}=\left(\begin{array}{cccc}
\sigma_{q_{1} q_{1}} & \sigma_{q_{1} q_{2}} & \sigma_{q_{1} p_{1}} & \frac{1}{\lambda} \sigma_{q_{1} p_{2}} \\
\sigma_{q_{2} q_{1}} & \sigma_{q_{2} q_{2}} & \sigma_{q_{2} p_{1}} & \frac{1}{\lambda} \sigma_{q_{2} p_{2}} \\
\sigma_{p_{1} q_{1}} & \sigma_{p_{1} q_{2}} & \sigma_{p_{1} p_{1}} & \frac{1}{\lambda} \sigma_{p_{1} p_{2}} \\
\frac{1}{\lambda} \sigma_{p_{2} q_{1}} & \frac{1}{\lambda} \sigma_{p_{2} q_{2}} & \frac{1}{\lambda} \sigma_{p_{2} p_{1}} & \frac{1}{\lambda^{2}} \sigma_{p_{2} p_{2}}
\end{array}\right) .
$$

We obtain a test of separability based on the Robertson-Schrödinger uncertainty relation for the partially scaled matrix $\sigma_{\lambda}$

$$
\operatorname{det}\left(\sigma_{\lambda}+\frac{i}{2} \Omega\right) \geq 0
$$




\section{Scaling Transform in the Three-Mode Case}

Changing the sign for one momentum only may not be efficient in the case of three and more modes. The Peres-Horodecki criterion detects the bipartite entanglement. This limitation can be overcome using the scaling transform by changing momenta for several modes in different ways. In the three-mode case, we can apply the following transform:

$$
\left\{\begin{array}{l}
p_{1} \longrightarrow \lambda_{1} p_{1}, \\
p_{2} \longrightarrow \lambda_{2} p_{2}, \\
p_{3} \longrightarrow \lambda_{3} p_{3} .
\end{array}\right.
$$

The scaling parameters are determined, as in the previous case, in the domain from -1 to 1 , namely, $\lambda_{1} \in[-1,1], \lambda_{2} \in[-1,1]$, and $\lambda_{3} \in[-1,1]$. The applied transform leads to the scaled variance matrix

$$
\sigma_{\lambda_{1} \lambda_{2} \lambda_{3}}=\left(\begin{array}{cccccc}
\sigma_{q_{1} q_{1}} & \sigma_{q_{1} q_{2}} & \sigma_{q_{1} q_{3}} & \frac{1}{\lambda_{1}} \sigma_{q_{1} p_{1}} & \frac{1}{\lambda_{2}} \sigma_{q_{1} p_{2}} & \frac{1}{\lambda_{3}} \sigma_{q_{1} p_{3}} \\
\sigma_{q_{2} q_{1}} & \sigma_{q_{2} q_{2}} & \sigma_{q_{2} q_{3}} & \frac{1}{\lambda_{1}} \sigma_{q_{2} p_{1}} & \frac{1}{\lambda_{2}} \sigma_{q_{2} p_{2}} & \frac{1}{\lambda_{3}} \sigma_{q_{2} p_{3}} \\
\sigma_{q_{3} q_{1}} & \sigma_{q_{3} q_{2}} & \sigma_{q_{3} q_{3}} & \frac{1}{\lambda_{1}} \sigma_{q_{3} p_{1}} & \frac{1}{\lambda_{2}} \sigma_{q_{3} p_{2}} & \frac{1}{\lambda_{3}} \sigma_{q_{3} p_{3}} \\
\frac{1}{\lambda_{1}} \sigma_{p_{1} q_{1}} & \frac{1}{\lambda_{1}} \sigma_{p_{1} q_{2}} & \frac{1}{\lambda_{1}} \sigma_{p_{1} q_{3}} & \frac{1}{\lambda_{1}^{2}} \sigma_{p_{1} p_{1}} & \frac{1}{\lambda_{1} \lambda_{2}} \sigma_{p_{1} p_{2}} & \frac{1}{\lambda_{1} \lambda_{3}} \sigma_{p_{1} p_{3}} \\
\frac{1}{\lambda_{2}} \sigma_{p_{2} q_{1}} & \frac{1}{\lambda_{2}} \sigma_{p_{2} q_{2}} & \frac{1}{\lambda_{2}} \sigma_{p_{2} q_{3}} & \frac{1}{\lambda_{1} \lambda_{2}} \sigma_{p_{2} p_{1}} & \frac{1}{\lambda_{2}^{2}} \sigma_{p_{2} p_{2}} & \frac{1}{\lambda_{2} \lambda_{3}} \sigma_{p_{2} p_{3}} \\
\frac{1}{\lambda_{3}} \sigma_{p_{3} q_{1}} & \frac{1}{\lambda_{3}} \sigma_{p_{3} q_{2}} & \frac{1}{\lambda_{3}} \sigma_{p_{3} q_{3}} & \frac{1}{\lambda_{1} \lambda_{3}} \sigma_{p_{3} p_{1}} & \frac{1}{\lambda_{2} \lambda_{3}} \sigma_{p_{3} p_{2}} & \frac{1}{\lambda_{3}^{2}} \sigma_{p_{3} p_{3}}
\end{array}\right) .
$$

The uncertainty relation for the scaled matrix demonstrates the criterion of separability. Since the first three principal minors remain unchanged, taking into consideration the initial uncertainty relation, the criterion reduces to the three inequalities:

$$
\begin{aligned}
& \Sigma\left(\lambda_{1}, \lambda_{2}, \lambda_{3}\right)=\operatorname{det}\left[\sigma_{\lambda_{1} \lambda_{2} \lambda_{3}}+\frac{i}{2}\left(\begin{array}{cc}
0 & -I \\
I & 0
\end{array}\right)\right] \geq 0, \\
& \Sigma_{5}\left(\lambda_{1}, \lambda_{2}\right)=\operatorname{det} \mathbf{M}_{5}\left[\sigma_{\lambda_{1} \lambda_{2} \lambda_{3}}+\frac{i}{2}\left(\begin{array}{cc}
0 & -I \\
I & 0
\end{array}\right)\right] \geq 0,
\end{aligned}
$$

and

$$
\Sigma_{3}\left(\lambda_{1}\right)=\operatorname{det} \mathbf{M}_{4}\left[\sigma_{\lambda_{1} \lambda_{2} \lambda_{3}}+\frac{i}{2}\left(\begin{array}{cc}
0 & -I \\
I & 0
\end{array}\right)\right] \geq 0
$$

Here $I$ is the $3 \times 3$ identity matrix. The fifth and fourth principal minors are denoted as $\mathbf{M}_{5}$ and $\mathbf{M}_{4}$, respectively. The criterion consists in checking the sign of functions $\Sigma\left(\lambda_{1}, \lambda_{2}, \lambda_{3}\right), \Sigma_{5}\left(\lambda_{1}, \lambda_{2}\right)$, and $\Sigma_{4}\left(\lambda_{1}\right)$ in the domain $\lambda_{1} \times \lambda_{2} \times \lambda_{3}=[-1,1] \times[-1,1] \times[-1,1]$. The negativity of one function is an indicator of entanglement of the given state with a certain variance matrix. 
Below it will be shown that the absolute value of negative function $\Sigma\left(\lambda_{1}, \lambda_{2}, \lambda_{3}\right)$ is connected with the degree of entanglement.

We consider a specific pure three-mode Gaussian state with the wave function as follows:

$$
\Psi(x, y, z)=N \exp \left(-\frac{x^{2}}{2}-\frac{y^{2}}{2}-\frac{z^{2}}{2}+c_{1} x y+c_{2} x z+c_{3} y z\right),
$$

where $c_{1}, c_{2}$, and $c_{3}$ are the parameters of quadratic form determining the degree of entanglement. The normalization constant $N$ reads

$$
N=\frac{\sqrt[4]{1-c_{1}^{2}-c_{2}^{2}-c_{3}^{2}-2 c_{1} c_{2} c_{3}}}{\pi^{3 / 4}} .
$$

In this case, the variance matrix $\sigma_{r_{i} r_{j}}$ can be calculated using the definition (10). After calculating the elements of $\sigma$, we obtain the following result:

$$
\sigma=\frac{1}{2}\left(\begin{array}{cc}
U & 0 \\
0 & V
\end{array}\right)
$$

where we use the notation

$$
\begin{aligned}
& U=\left(1-c_{1}^{2}-c_{2}^{2}-c_{3}^{2}-2 c_{1} c_{2} c_{3}\right)^{-1}\left(\begin{array}{ccc}
1-c_{3}^{2} & c_{1}+c_{2} c_{3} & c_{2}+c_{1} c_{3} \\
c_{1}+c_{2} c_{3} & 1-c_{2}^{2} & c_{3}+c_{1} c_{2} \\
c_{2}+c_{1} c_{3} & c_{3}+c_{1} c_{2} & 1-c_{1}^{2}
\end{array}\right) \\
& V=\left(\begin{array}{ccc}
1 & -c_{1} & -c_{2} \\
-c_{1} & 1 & -c_{3} \\
-c_{2} & -c_{3} & 1
\end{array}\right)
\end{aligned}
$$

The uncertainty relations for the state under study and the normalization conditions determine the admissible domain of the coefficients of the quadratic form. The following relations show the possible domain of the coefficients:

$$
\left\{\begin{array}{l}
c_{1} \in(-1,1) \\
c_{2} \in(-1,1) \\
c_{3} \in(-1,1) \\
1-c_{1}^{2}-c_{2}^{2}-c_{3}^{2}-2 c_{1} c_{2} c_{3}>0
\end{array}\right.
$$

The Peres-Horodecki criterion for the three-mode case is obtained by applying the transform of quadratures as follows:

$$
\left\{\begin{array}{l}
p_{1} \longrightarrow p_{1} \\
p_{2} \longrightarrow-p_{2} \\
p_{3} \longrightarrow-p_{3}
\end{array}\right.
$$

The Robertson-Schrödinger uncertainty relation for the transformed matrix provides two inequalities - one from determinants of the whole variance matrix and the other from the fifth principal 

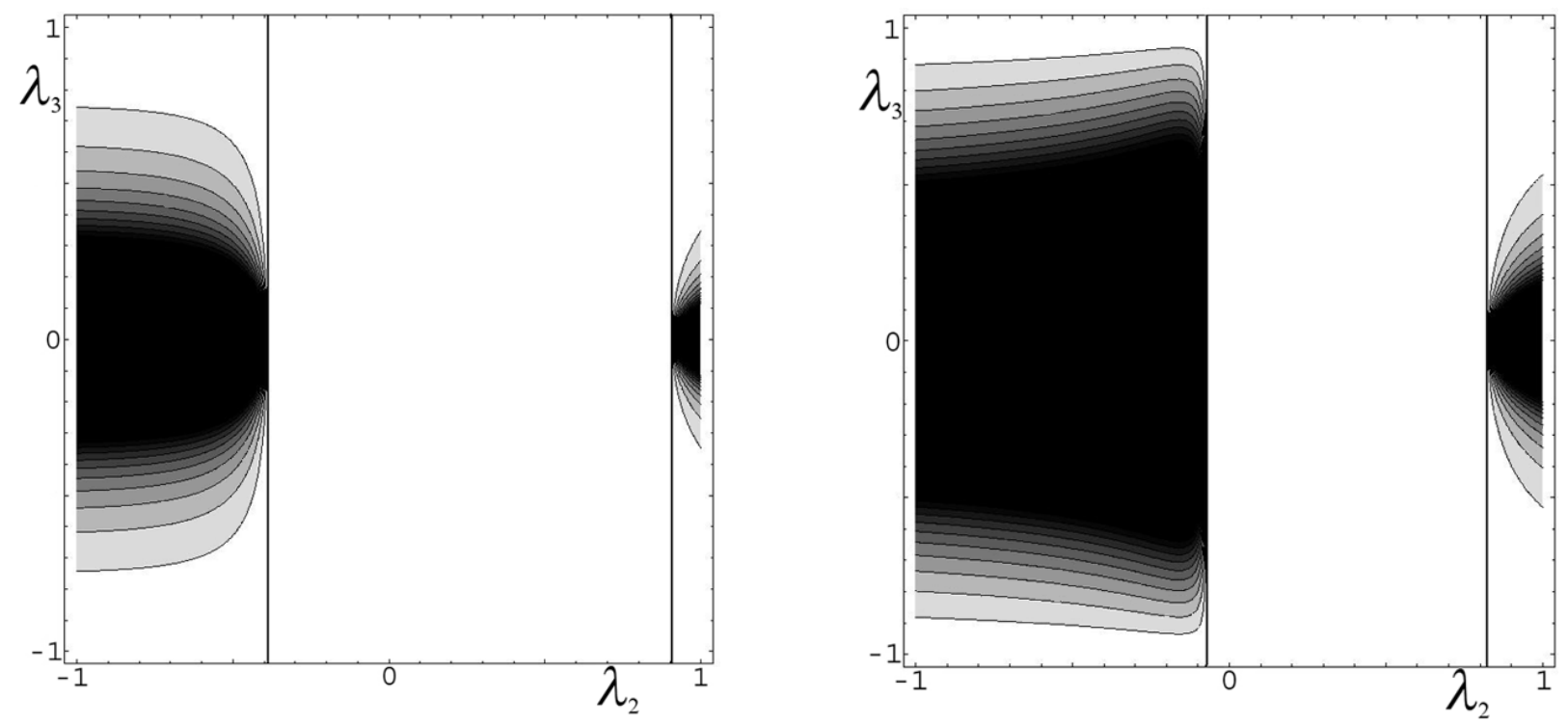

Fig. 1. Contour plots of $\Sigma\left(\lambda_{1}, \lambda_{2}, \lambda_{3}\right)$ for the wave function (36), where $\lambda_{1}=1 / 2$ and $c_{1}=2 / 3$ (on the left) and $c_{1}=5 / 6$ (on the right).

minor of the matrix. The determinant [analogous to (24) in the three-mode case] is equal to zero for any $c_{1}, c_{2}$, and $c_{3}$. The determinant of the fifth principal minor is proportional to $c_{1}^{2}$, which leads to the conclusion that the Peres-Horodecki criterion does not detect any entanglement for all wave functions of the form (30) with $c_{1}=0$.

Below we examine the application of the partial scaling criterion to the wave function (30) for certain coefficients $c_{1}, c_{2}$, and $c_{3}$.

First, we consider the case where the entanglement is given only by the term $x y$ or, in the language of coefficients, the case $c_{2}=c_{3}=0$. Obviously, the problem is symmetric with respect to permutations of $c_{1}, c_{2}$ and $c_{3}$. From relations (30) and (31) and conditions (34), we have the following form of wave function for any $c_{1} \in(-1,1)$

$$
\Psi(x, y, z)=\frac{\sqrt[4]{1-c_{1}^{2}}}{\pi^{3 / 4}} \exp \left(-\frac{x^{2}}{2}-\frac{y^{2}}{2}-\frac{z^{2}}{2}+c_{1} x y\right) .
$$

Simplifying the variance matrix (32) and applying the partial scaling transform (25) we obtain

$$
\Sigma\left(\lambda_{1}, \lambda_{2}, \lambda_{3}\right)=-\frac{1}{64 \lambda_{1}^{2} \lambda_{2}^{2} \lambda_{3}^{2}} \frac{c_{1}^{2}\left(1-\lambda_{1} \lambda_{2}\right)^{2}-\left(1-\lambda_{1}^{2}\right)\left(1-\lambda_{2}^{2}\right)}{1-c_{1}^{2}} .
$$

For $c_{1}=0$, expression (37) is nonnegative in the domain $\lambda_{1} \times \lambda_{2} \times \lambda_{3}=[-1,1] \times[-1,1] \times$ $[-1,1]$. So, for this separable state, the uncertainty relation holds and the criterion does not show any entanglement. Otherwise, for any $c_{1} \in(-1,1)$, such a pair $\lambda_{1}$ and $\lambda_{2}$ exists for which $\Sigma\left(\lambda_{1}, \lambda_{2}, \lambda_{3}\right)$ is negative. Thus, the partial scaling criterion (27) detects the entanglement in this case. Moreover, it is easy to see that the rate and area of negativity increases with the coefficient $c_{1}$. These facts will be demonstrated by some examples below. 

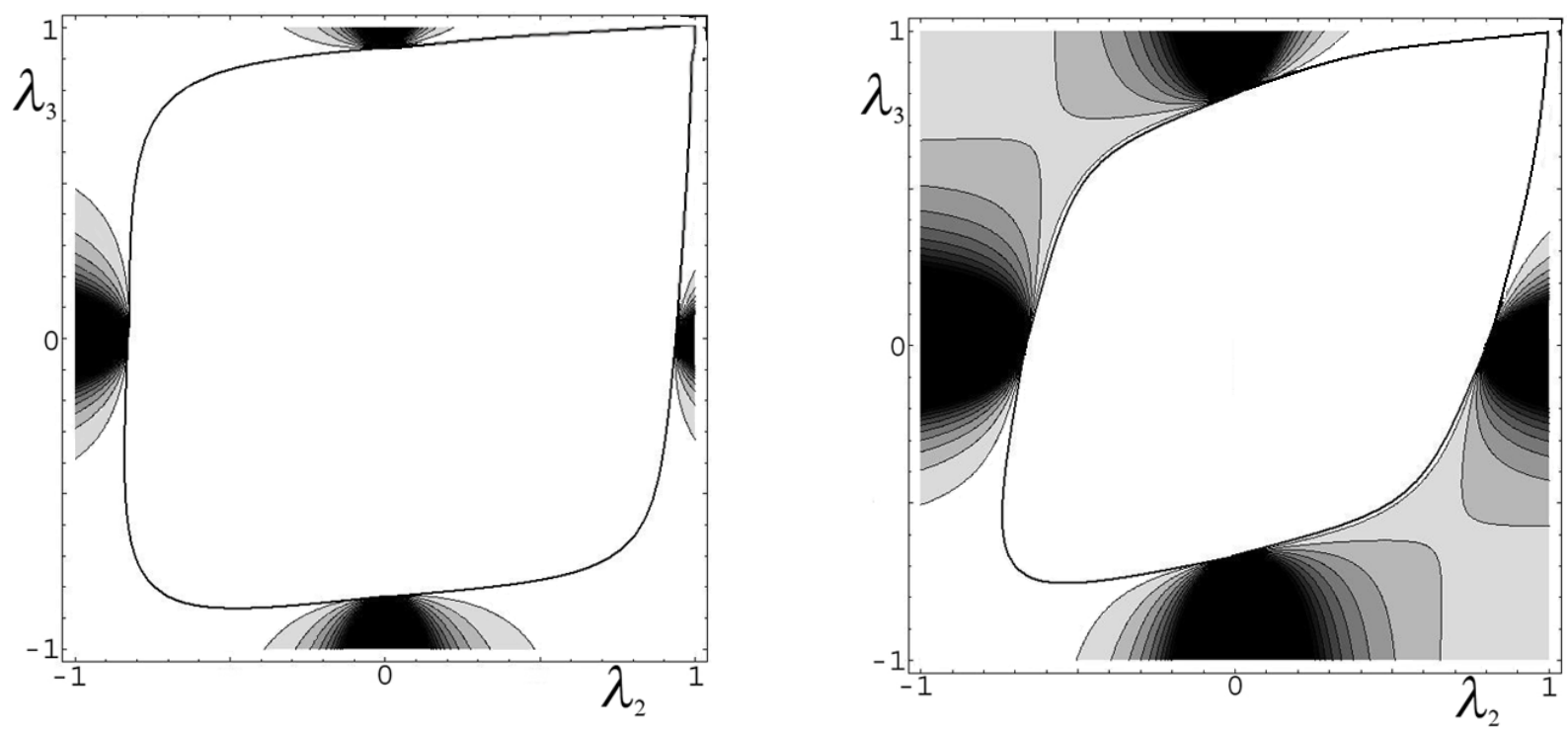

Fig. 2. Contour plots of $\Sigma\left(\lambda_{1}, \lambda_{2}, \lambda_{3}\right)$ for $c_{1}=c_{2}=1 / 4$, where $\lambda_{1}=1 / 2$ and $c_{3}=1 / 4$ (on the left) and $c_{3}=1 / 2$ (on the right).

An illustrative example is shown in Fig. 1. Contour plots present the function $\Sigma\left(\lambda_{1}, \lambda_{2}, \lambda_{3}\right)$ with $\lambda_{1}=1 / 2$. To show that the graph is moving down with increase in entanglement, we constructed contour plots for $c_{1}=2 / 3$ and $5 / 6$. The negative area of the graph for bigger coefficient $c_{1}$ is larger and lower than for smaller $c_{1}$. Since we are not interested in the positive part of the graphs, the grey scale shows only the rate of negativity. The negative part of the graphs is bounded by lines, where the function is equal to zero. The area between lines is a positive part of the function. It is worth mentioning that for $c_{1} \rightarrow 1$ or, in other words, for the maximum entanglement in this case, the positive part collapses to the line $\lambda_{2}=\lambda_{1}$. In the considered case, it is the line $\lambda_{2}=1 / 2$.

The completely entangled state leads to the

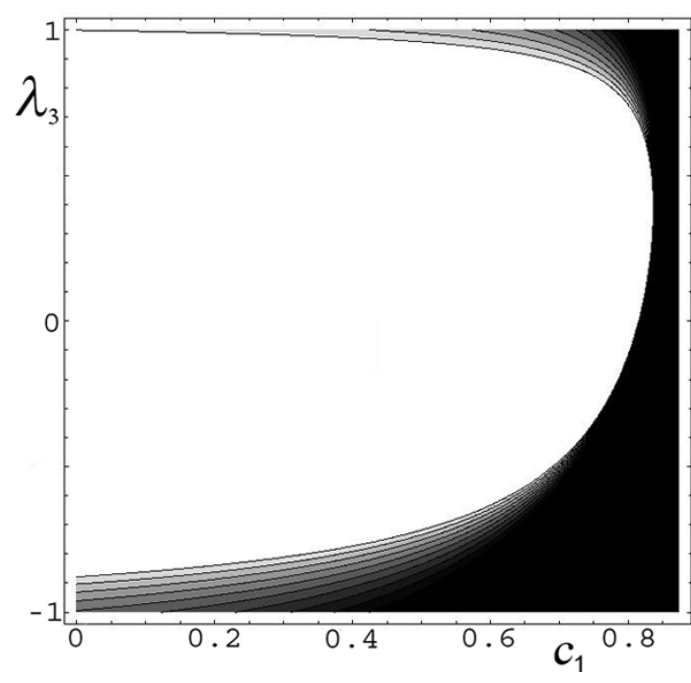

Fig. 3. Contour plot of $\Sigma\left(\lambda_{1}, \lambda_{2}, \lambda_{3}\right)$ for $c_{2}=c_{3}=1 / 4$, where $\lambda_{1}=1 / 2, \lambda_{2}=1 / 4$. same result. We demonstrate it by the case $c_{1}=c_{2}=1 / 4$. In Fig. 2 graphs for $c_{3}=1 / 4$ and $1 / 2$ are shown. Obviously, the graph for bigger $c_{3}$ has larger negative area and its absolute value in points, where both functions are negative, is bigger. The zero level is shown by a black curve. The area inside it contains only positive values.

A vivid illustration that the graph is mowing down with increase in entanglement is shown in Fig. 3 where the function is constructed for the wave function with $c_{2}=c_{3}=1 / 4$. As before, it 
represents $\Sigma\left(\lambda_{1}, \lambda_{2}, \lambda_{3}\right)$ with $\lambda_{1}=1 / 2$ and $\lambda_{2}=1 / 4$. Obviously, the positive part of plot (white color) diminishes with increase in $c_{1}$ from 0 to $7 / 8$ and the negative part is moving down.

\section{Scaling Transform in the Four-Mode Case}

To apply the graphing method discussed in the previous sections to the four-mode state, we apply the transform of momenta of the form

$$
\left\{\begin{array}{l}
p_{1} \longrightarrow \lambda_{1} p_{1} \\
p_{2} \longrightarrow \lambda_{2} p_{2} \\
p_{3} \longrightarrow \lambda_{3} p_{3} \\
p_{4} \longrightarrow \lambda_{4} p_{4}
\end{array}\right.
$$
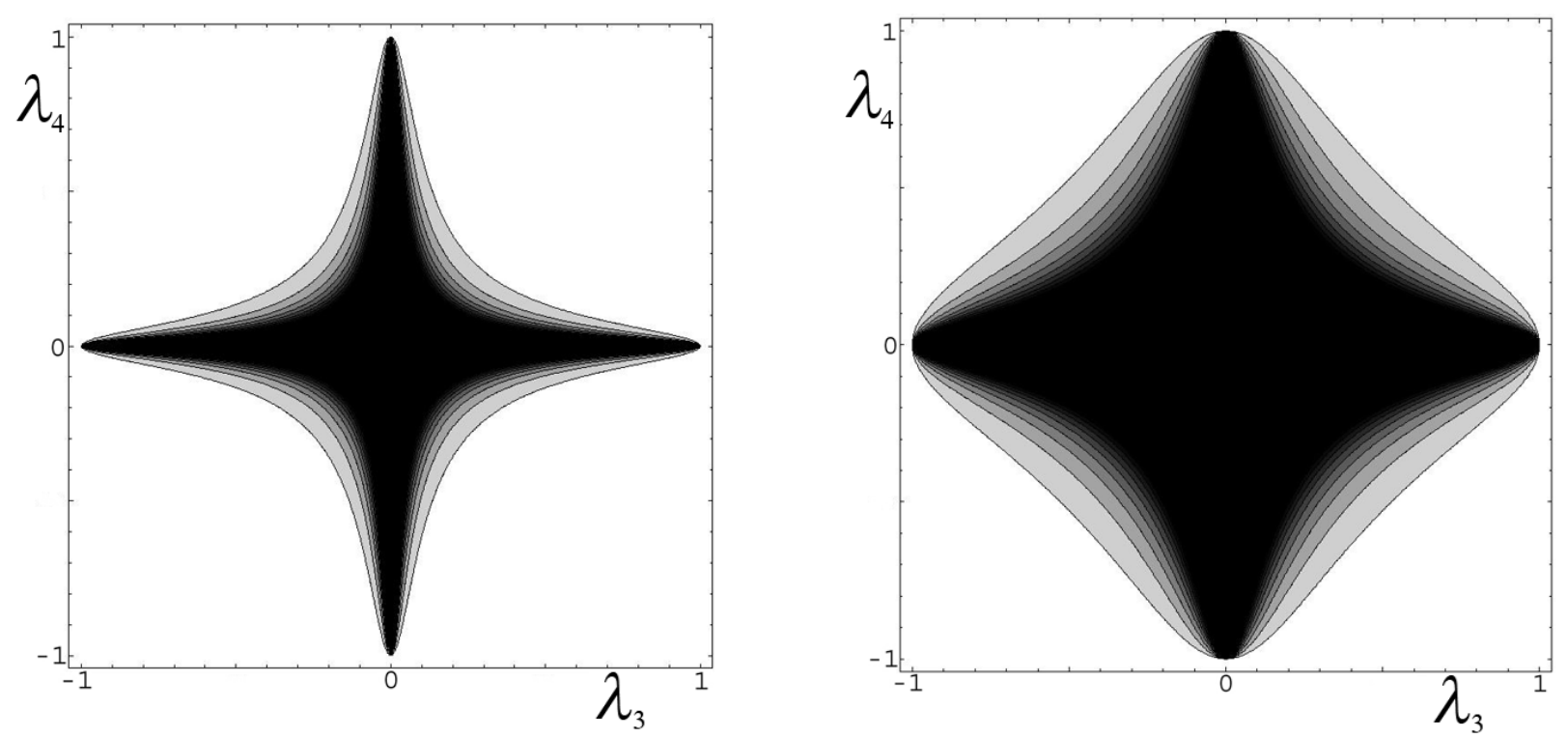

Fig. 4. Contour plots of $\Sigma\left(\lambda_{1}, \lambda_{2}, \lambda_{3}, \lambda_{4}\right)$ for the wave function (40), where $\lambda_{1}=-1$ and $\lambda_{2}=1 / 2$ and $c_{1}=1 / 8$ (on the left) and $c_{1}=1 / 2$ (on the right).

All scaling parameters belong to the interval $[-1,1]$. The transformed variance $8 \times 8$ matrix $\sigma_{\lambda_{1} \lambda_{2} \lambda_{3} \lambda_{4}}$ is similar to (26). The Robertson-Schrödinger uncertainty relation can be easily reduced to four inequalities, in complete analogy to the previous case. We consider only the inequality for determinant of this matrix. It will be shown that the function defined below gives results very similar to the ones obtained in the three-mode case. The inequality under consideration reads

$$
\Sigma\left(\lambda_{1}, \lambda_{2}, \lambda_{3}, \lambda_{4}\right)=\operatorname{det}\left[\sigma_{\lambda_{1} \lambda_{2} \lambda_{3} \lambda_{4}}+\frac{i}{2}\left(\begin{array}{cc}
0 & -I \\
I & 0
\end{array}\right)\right] \geq 0
$$

It is worth mentioning that the Peres-Horodecki criterion applied to the four-mode wave function does not give complete information on entanglement - in some cases, it does not work. 

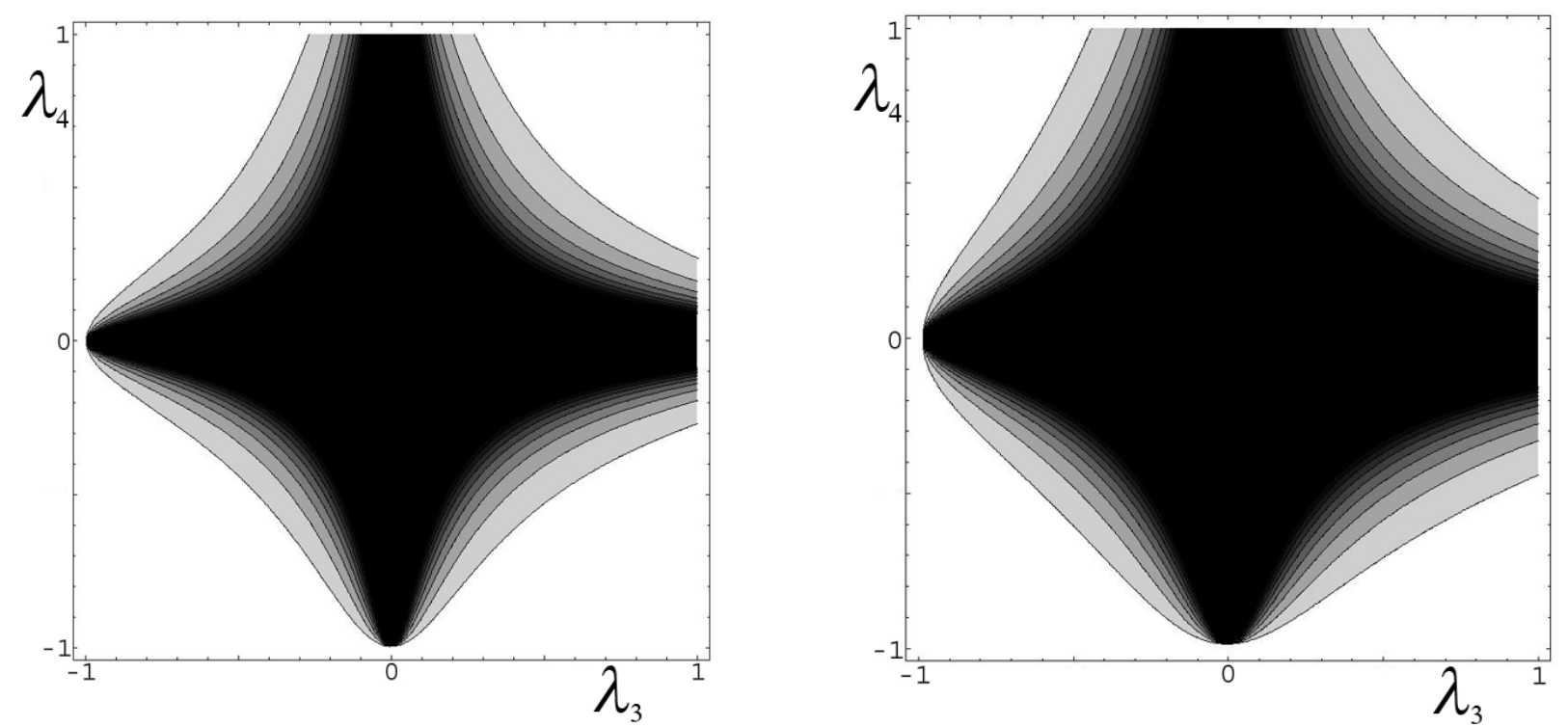

Fig. 5. Contour plots of $\Sigma\left(\lambda_{1}, \lambda_{2}, \lambda_{3}, \lambda_{4}\right)$ for the wave function (42), where $\lambda_{1}=-1$ and $\lambda_{2}=1 / 2$ and $c_{6}=1 / 8$ (on the left) and $c_{6}=1 / 2$ (on the right).

We investigate wave functions of the form analogous to the ones considered in the three-mode case. First, let us look at the function

$$
\Psi(x, y, z, k)=N \exp \left(-\frac{x^{2}}{2}-\frac{y^{2}}{2}-\frac{z^{2}}{2}-\frac{k^{2}}{2}+c_{1} x y\right),
$$

where

$$
N=\frac{\sqrt[4]{1-c_{1}^{2}}}{\pi}
$$

Graphs of $\Sigma\left(-1,1 / 2, \lambda_{3}, \lambda_{4}\right)$ for $c_{1}=1 / 8$ and $1 / 2$ are shown in Fig. 4. One can observe the same effect of moving the graph down with increase in entanglement. Entire graphs are located in the nonpositive half-space.

For completeness, in Fig. 5 contour plots for completely entangled states are shown. The graphs are constructed for

$$
\Psi(x, y, z, k)=N \exp \left(-\frac{x^{2}}{2}-\frac{y^{2}}{2}-\frac{z^{2}}{2}-\frac{k^{2}}{2}+\frac{1}{4} x y+\frac{1}{4} x z+\frac{1}{4} x k+\frac{1}{4} y z+\frac{1}{4} y k+c_{6} z k\right),
$$

where $c_{6}=1 / 8$ and $1 / 2$.

In both cases, the partial scaling transform detects the entanglement. Also it can be seen that the bigger the entanglement, the lower the location of the plot of $\Sigma\left(\lambda_{1}, \lambda_{2}, \lambda_{3}, \lambda_{4}\right)$. The two functions are nonpositive in the domain $\lambda_{3} \times \lambda_{4}=[-1,1] \times[-1,1]$ too. 


\section{Scaling Transform Criterion for Mixed Gaussian States}

In this section, the partial scaling method is tested on the applicability for mixed Gaussian states. First, we consider the three-mode state with the variance matrix

$$
\sigma=\left(\begin{array}{cccccc}
\frac{6}{5} & \frac{1}{5} & \frac{1}{5} & \frac{1}{10} & \frac{1}{10} & \frac{1}{10} \\
\frac{1}{5} & \frac{6}{5} & \frac{1}{5} & \frac{1}{10} & \frac{1}{10} & \frac{1}{10} \\
\frac{1}{5} & \frac{1}{5} & \frac{6}{5} & \frac{1}{10} & \frac{1}{10} & \frac{1}{10} \\
\frac{1}{10} & \frac{1}{10} & \frac{1}{10} & \frac{1}{2} & -\frac{1}{8} & -\frac{1}{8} \\
\frac{1}{10} & \frac{1}{10} & \frac{1}{10} & -\frac{1}{8} & \frac{1}{2} & -\frac{1}{8} \\
\frac{1}{10} & \frac{1}{10} & \frac{1}{10} & -\frac{1}{8} & -\frac{1}{8} & \frac{1}{2}
\end{array}\right) .
$$

The graph for $\Sigma\left(1, \lambda_{2}, \lambda_{3}\right)$ is presented in Fig. 6 (on the left) where the positive part of the graph is shown with white color. Obviously, the partial scaling method detects the entanglement in this case - the graph has a negative area in the domain $\lambda_{2} \times \lambda_{3}=[-1,1] \times[-1,1]$.
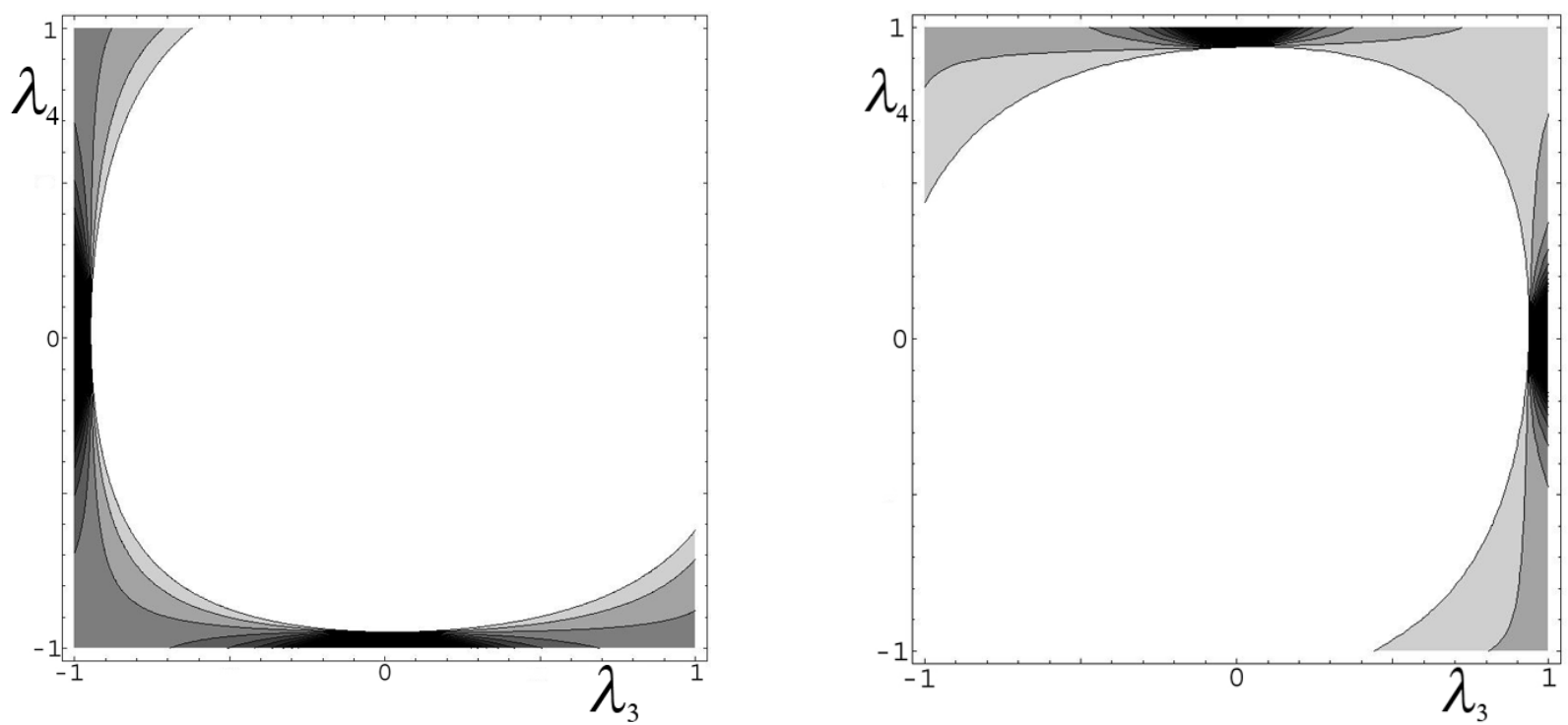

Fig. 6. Contour plot of $\Sigma\left(\lambda_{1}, \lambda_{2}, \lambda_{3}\right)$ for the variance matrix (43), where $\lambda_{1}=1$ (on the left) and contour plot of $\Sigma\left(\lambda_{1}, \lambda_{2}, \lambda_{3}, \lambda_{4}\right)$ for the variance matrix (44), where $\lambda_{1}=-1$ and $\lambda_{2}=1 / 2$ (on the right).

A similar result is obtained for the four-mode mixed Gaussian state. In Fig. 6 (on the right) the graph for $\Sigma\left(-1,1 / 2, \lambda_{3}, \lambda_{4}\right)$ is shown. 
The $8 \times 8$ variance matrix reads

$$
\sigma=\left(\begin{array}{cccccccc}
\frac{8}{5} & \frac{2}{5} & \frac{2}{5} & \frac{2}{5} & \frac{1}{10} & \frac{1}{10} & \frac{1}{10} & \frac{1}{10} \\
\frac{2}{5} & \frac{8}{5} & \frac{2}{5} & \frac{2}{5} & \frac{1}{10} & \frac{1}{10} & \frac{1}{10} & \frac{1}{10} \\
\frac{2}{5} & \frac{2}{5} & \frac{8}{5} & \frac{2}{5} & \frac{1}{10} & \frac{1}{10} & \frac{1}{10} & \frac{1}{10} \\
\frac{2}{5} & \frac{2}{5} & \frac{2}{5} & \frac{8}{5} & \frac{1}{10} & \frac{1}{10} & \frac{1}{10} & \frac{1}{10} \\
\frac{1}{10} & \frac{1}{10} & \frac{1}{10} & \frac{1}{10} & \frac{1}{2} & -\frac{1}{8} & -\frac{1}{8} & -\frac{1}{8} \\
\frac{1}{10} & \frac{1}{10} & \frac{1}{10} & \frac{1}{10} & -\frac{1}{8} & \frac{1}{2} & -\frac{1}{8} & -\frac{1}{8} \\
\frac{1}{10} & \frac{1}{10} & \frac{1}{10} & \frac{1}{10} & -\frac{1}{8} & -\frac{1}{8} & \frac{1}{2} & -\frac{1}{8} \\
\frac{1}{10} & \frac{1}{10} & \frac{1}{10} & \frac{1}{10} & -\frac{1}{8} & -\frac{1}{8} & -\frac{1}{8} & \frac{1}{2}
\end{array}\right) .
$$

In both plots, there is a negative area, which depicts the entanglement of states with the variance matrices (43) and (44).

\section{Conclusions}

To conclude, we point out the main results of our work.

We have shown that the new criterion of entanglement based on the scaling transform of the photon quadrature components in the Wigner function of the quantum state provides the possibility to detect the entanglement for three- and four-mode fields. The calculated principal minors of the scaled quadrature dispersion matrices shifted by the quadrature commutator matrix become negative for entangled Gaussian states. The values of modulus of the negative principal minors can serve as empirical characteristics of the degree of entanglement. The results obtained for the three-mode entangled Gaussian states, in general, agree with the previous results of [13, 14] where the scaling criterion of entanglement was applied to the photon states created due to the light interaction with nonlinear crystal. In a future publication, we will extend the analysis of the entanglement based on the scaling criterion to the multimode light beams (both of Gaussian and non-Gaussian tipes) and compare this criterion with other approaches to the problem of detecting the entanglement.

\section{Acknowledgments}

V.I.M. acknowledges the support of the Russian Foundation for Basic Research under Project No. 07-02-00598. 


\section{References}

1. R. Simon, Phys. Rev. Lett., 84, 2726 (2000).

2. L.-M. Duan, G. Giedke, J. I. Cirac, and P. Zoller, Phys. Rev. Lett., 84, 2722 (2000).

3. A. Peres, Phys. Rev. Lett., 77, 1413 (1996).

4. P. Horodecki, R. Horodecki, and M. Horodecki, Phys. Lett. A, 223, 1 (1996).

5. E. C. G. Sudarshan, R. J. Mathews, and J. Rau, Phys. Rev., 121, 920 (1961).

6. E. Shchukin and W. Vogel, Phys. Rev. Lett., 95, 230502 (2005).

7. E. Shchukin and W. Vogel, Phys. Rev. Lett., 96, 200403 (2006).

8. E. Shchukin and W. Vogel, Phys. Rev. A, 74, 030302 (2006).

9. G. Adesso, A. Serafini, and F. Illuminati, Phys. Rev. A, 73, 032345 (2006).

10. O. V. Man'ko, V. I. Man'ko, G. Marmo, et al., Phys. Lett. A, 339, 194 (2005).

11. O. V. Man'ko, V. I. Man'ko, G. Marmo, et al., Phys. Lett. A, 357, 255 (2006).

12. E. Wigner, Phys. Rev., 40, 749 (1932).

13. A. S. Chirkin and M. Yu. Saigin, Acta Phys. Hung. B, 26/1-2, 63 (2006).

14. M. Yu. Saigin and A. S. Chirkin, Mod. Probl. Stat. Phys., 5, 169 (2006).

15. E. Schrödinger, Ber. Kgl. Akad. Wiss. Berlin, 24, 296 (1930).

16. H. P. Robertson, Phys. Rev., 35, 667 (1930). 